\title{
Oxyradical Mediated Tissue Injury
}

Jeffrey S. Warren, M.D.

Peter A. Ward, M.D.

Department of Pathology

University of Michigan Medical School

Ann Arbor, Michigan

Since the beginning of this century, phagocytes have been recognized for their critical role in the inflammatory response. In addition to their functions in host defense, phagocytes (neutrophils, monocytes, and macrophages) can mediate tissue injury through a variety of mechanisms (3). Traditionally, attention has been focused on the potential for leukocytic lysosomal proteases to damage tissue. In recent years, it has been recognized that lysosomal enzymes cannot completely account for the tissue-damaging effects of phagocytes. Recent studies have provided compelling evidence that oxygen-derived free radicals are important mediators of tissue injury. Free radicals are atoms or molecules that possess unpaired electrons. Through a variety of reactions, oxygen free radicals can participate in the generation of additional reactive oxygen-derived metabolites. The focus of this article will be how phagocytes, particularly polymorphonuclear leukocytes (PMN), generate these substances. We will examine the in vitro and in vivo evidence that oxygen radicals and their metabolites are important mediators of tissue injury.

\section{Production of Oxyradicals by Phagocytes}

After membrane perturbation by spe- cific soluble or particulate stimuli, phagocytes release their lysosomal contents and exhibit a 2-20-fold increase in oxygen consumption $(1,13)$. The increase in oxygen consumption is associated with activation of the hexose monophosphate shunt and generation of a variety of oxygen-derived metabolites. Whereas the principal oxygenderived species are superoxide anion $\left(\mathrm{O}_{\overline{2}}\right)$ and hydrogen peroxide $\left(\mathrm{H}_{2} \mathrm{O}_{2}\right)$, additional chemical reactions give rise to a host of reactive oxidants (Table 1). All of these oxygen-derived substances are thought to have roles in tissue injury and modulation of the inflammatory response.

The plasma membrane-associated NADPH oxidase system appears to be the most important source of phagocyte-derived $\mathrm{O}_{\overline{2}}^{-}$. Despite the general reactivity of free radicals, $\mathrm{O}_{\overline{2}}^{-}$is relatively nonreactive in aqueous media, especially at neutral $\mathrm{pH}(20)$. Either spontaneously or with the aid of a group of metalloenzymes called superoxide dismutases (SOD), $\mathrm{O}_{\overline{2}}$ can undergo dismutation to $\mathrm{H}_{2} \mathrm{O}_{2}(5)$. Unlike $\mathrm{O}_{\overline{2}}$, hydrogen peroxide is a powerful oxidant. Capable of directly oxidizing a variety of biologic molecules, $\mathrm{H}_{2} \mathrm{O}_{2}$ can also participate in chemical reactions resulting in generation of additional oxidants (Table 1). Two of the most important metabolic products of $\mathrm{H}_{2} \mathrm{O}_{2}$ are the hydroxyl radical (HO) and myeloperoxidase-halide generated substances such as hypochlorous acid ( $\mathrm{HOCl})$. The hydroxyl radical is a potent oxidizing agent that can react with numerous organic and inorganic com- pounds (23). Much of the evidence that phagocytes or phagocyte-derived substances can generate $\mathrm{HO}^{\circ}$ is indirect since it centers on studies which em-

\section{In This Issue}

\section{Oxyradical Mediated Tissue}

Injury $\ldots \ldots \ldots \ldots \ldots \ldots \ldots 113$

In vitro and in vivo evidence for the pathogenetic role of phagocytes with special reference to polymorphonuclear leukocytes

\section{Suppression of Human Natural Killer Activity by Delta-9. \\ Tetrahydrocannabinol .......116 Discussion of potential mechanisms responsible for this effect}

Immunohistochemistry of Human

Tumors .................119

Technical considerations about this ancillary tool for the study of "undifferentiated" or "problem" neoplasms

Synthetic Peptide Vaccines and the Prediction of Epitopes in Proteins .................122 Practical problems and how they may be solved prior to human use

AIDS Virus-Vaccine

Prospects ................125

Elucidation of the prerequisites, potential problems, and approaches for the development of effective vaccines 
ploy HO scavengers or measurement of $\mathrm{HO}^{\circ}$ oxidation products. Monocytes and neutrophils contain myeloperoxidases which are heme containing enzymes found in primary lysosomal granules (17). Myeloperoxidases can catalyze the oxidation of halides by $\mathrm{H}_{2} \mathrm{O}_{2}$ to form hypohalous acids such as $\mathrm{HOCl}$ which can oxidize a large variety of biologic molecules. In addition, biologic molecules can be directly halogenated by phagocyte-derived peroxidases and $\mathrm{H}_{2} \mathrm{O}_{2}$.

The precise mechanisms by which oxygen-derived substances inflict cell and tissue injury are unclear but appear to involve membrane lipid peroxidation reactions and direct protein or carbohydrate modifications $(3,4)$.

\section{In Vitro Evidence of Oxyradical Mediated Toxicity}

Numerous in vitro studies have shown that oxygen-derived metabolites can mediate toxicity involving a variety of biomolecules and eukaryotic cells. Following the observation that $\mathrm{O}_{\dot{2}}^{-}$will depolymerize hyaluronic acid, several studies have demonstrated the ability of oxyradicals to inactivate or modify biologically relevant molecules including collagen, various enzymes, $\alpha$-1-antiprotease, and arachidonate-derived lipids (6). These studies provide evidence that oxygen metabolites can directly alter stromal tissue components (collagen, hyaluronic acid). Inactivation of $\alpha$ - 1 -antiprotease and generation of oxidized arachidonate-derived chemotactic lipids are examples of proinflammatory mechanisms attributable to oxyradicals $(2,15)$. There is an extensive body of data demonstrating that oxygen metabolites generated by PMNs, macrophages, or monocytes are directly cytotoxic to a variety of cells. Endothelial cells, fibroblasts, tumor cells, erythrocytes, platelets, and spermatozoa have all been found to be susceptible to oxygen-derived metabolites (3). It is clear that the mechanisms involved in oxyradical mediated cytotoxicity are complex and vary according to the stimulus, phagocytic cell type in-

Table 1

\section{Proposed Mechanisms of Formation of Oxygen-Derived Metabolites}

1. Reduction of molecular oxygen

$$
\mathrm{O}_{2}+\mathrm{e}^{-} \rightarrow \mathrm{O}_{2}^{-} \text {(superoxide anion) }
$$

2. Dismutation of superoxide anion

$$
2 \mathrm{O}_{2}^{\bar{z}}+2 \mathrm{H}^{+} \rightarrow \mathrm{H}_{2} \mathrm{O}_{2}+\mathrm{O}_{2} \text { (or ' } \mathrm{O}_{2} \text { ) (singlet oxygen) }
$$

3. Haber-Weiss reaction

$$
\mathrm{O}_{2}^{\bar{T}}+\mathrm{H}_{2} \mathrm{O}_{2} \rightarrow \mathrm{O}_{2} \text { (or ' } \mathrm{O}_{2} \text { ) }+\mathrm{HO}^{-}+\mathrm{HO}^{\circ} \text { (hydroxyl radical) }
$$

4. Fenton reaction

$$
\begin{gathered}
\mathrm{O}_{2}^{-}+\mathrm{Fe}^{3+} \rightarrow \mathrm{Fe}^{2+}+\mathrm{O}_{2} \\
\frac{\mathrm{Fe}^{2+}+\mathrm{H}_{2} \mathrm{O}_{2} \rightarrow \mathrm{Fe}^{3+}+\mathrm{HO}^{-}+\mathrm{HO}^{\circ}}{\mathrm{O}_{2}^{-}+\mathrm{H}_{2} \mathrm{O}_{2} \rightarrow \mathrm{O}_{2}+\mathrm{HO}^{-}+\mathrm{HO}^{\circ} \text { (hydroxyl radical) }}
\end{gathered}
$$

5. Myeloperoxidase - hydrogen peroxide - halide system

$$
\mathrm{H}_{2} \mathrm{O}_{2}+\mathrm{Cl}^{-} \stackrel{\text { myeloperoxidase }}{\longrightarrow} \mathrm{H}_{2} \mathrm{O}+\mathrm{HOCl} \text { (hypochlorous acid) }
$$

volved, oxygen-derived species produced, target cell susceptibility, and physicochemical milieu of the system. For example, erythrocyte destruction appears to be mediated by $\mathrm{O}_{2}^{-}$, which oxidizes hemoglobin after entering the cytoplasm through anionic channels, whereas some tumor cells are most sensitive to $\mathrm{HOCl}$ and endothelial cells are lysed by $\mathrm{H}_{2} \mathrm{O}_{2}$ or $\mathrm{HO}$ generated from $\mathrm{H}_{2} \mathrm{O}_{2}$ via the Fenton reaction (3, 21). Thus, there is extensive in vitro evidence that oxyradicals can damage both cells and stromal constituents.

\section{In Vivo Evidence of Oxyradical Mediated Tissue Injury}

A large number of in vivo models involving various organ systems have provided evidence that oxygen-derived metabolites are directly linked to the damaging of tissues. As in the in vitro systems, there is a variety of mechanisms that appear to be important depending on the initiating stimulus, organ system and phagocytic cell type.

There is in vivo evidence that ischemia-associated organ injury is related to the production of oxyradicals, either within affected tissues per se or by PMNs that have accumulated at the injury site after reperfusion. Transient ischemic injury to the small bowel of cats results in increased intestinal capillary permeability and albumin clearance (7). This increase in intestinal capillary permeability can be blocked by prior systemic administration of SOD or allopurinol. These data suggest that $\mathrm{O}_{2}^{-}$or other oxyradicals are mediators of ischemia-reperfusion injury. The mechanism of this injury appears to involve proteolytic conversion of hypoxanthine/xanthine dehydrogenase to hypoxanthine/xanthine oxidase within the cytoplasm of ischemic cells. When the ischemic tissue is reperfused, xanthine oxidase catalyzes the conversion of hypoxanthine and xanthine to uric acid. $\mathrm{O}_{\overline{2}}^{-}$and $\mathrm{H}_{2} \mathrm{O}_{2}$ are generated as byproducts of this reaction. Because allopurinol inhibits xanthine oxidase, uric acid and oxyradical formation are retarded, thereby protecting the tissue from oxidant injury.

In experimental myocardial infarcts, ischemia-reperfusion injury can be inhibited by SOD as well as by catalase. Ischemic myocardium contains far less xanthine oxidase activity than does intestinal tissue, suggesting that tissuederived oxidants may be less important mediators of injury in this model. This hypothesis is supported by the observa-

Clinical Immunology Newsletter (ISSN 0197-1859) is issued monthly in one indexed volume per year hy Elscvier Science Publishing Co.. Inc.. 52 Vanderbilt Avenue, New York, NY IOU17. Subseription price per

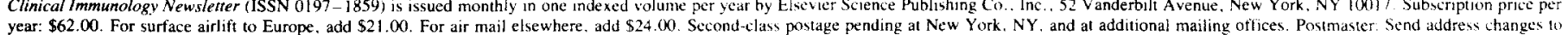
Clinical Immunology Newisletter. Elsevier Science Publishing Co.. Inc., 52 Vanderbilt Avenue. New York. NY IIn)17. 
tion that neutrophil depletion also affords protection of myocardium in ischemia-reperfusion (16). In catalasetreated animals there is reduction of myocardial injury, even though PMNs accumulate at the site of reperfusion. These two models of ischemia-reperfusion tissue injury illustrate oxyradicalmediated injury produced by apparently different mechanisms.

The role of $\mathrm{O}_{2}^{-}$in tissue injury has been examined in other in vivo models. These studies center largely on the ability of systemically administered SOD to suppress the development of tissue injury. SOD will inhibit edema formation in the acute immune complex-induced vasculitis or alveolitis, both of which reactions are neutrophil- and complement-dependent (14). In SOD-treated animals, there is a striking decrease in the number of neutrophils, suggesting that in this model SOD retards the influx of neutrophils rather than directly blocking injurious effects of $\mathrm{O}_{\dot{2}}^{-}$. These data corroborate other in vitro studies which demonstrate the ability of $\mathrm{O}_{2}^{-}$to generate arachidonate-derived lipids that are chemotactic for PMNs.

Much of the evidence implicating leukocyte-derived $\mathrm{H}_{2} \mathrm{O}_{2}$ in tissue injury has come from studies in which systemic administration of catalase suppresses tissue injury. Johnson et al. have described an acute immune complex lung injury model in which administration of catalase results in dosedependent suppression of lung injury (8). Histologic examination of catalase-protected animals shows that there is little lung cell injury, edema, or hemorrhage. In contrast to SODtreated animals, there were numerous neutrophils, suggesting that protective effects of catalase are mediated by removal of $\mathrm{H}_{2} \mathrm{O}_{2}$ rather than by prevention of influx of PMNs.

Two additional lung injury models involving vascular injury by neutrophil-derived oxyradicals have been described. In the first model, pulmonary vascular injury is initiated by cobra venom factor-induced activation of complement (18). The resulting lung injury is dependent on neutrophils and can be largely suppressed by catalase and to a lesser extent by SOD. This lung injury is associated with pulmonary capillary endothelial cell damage and edema. The second vascular lung injury model involves systemic complement activation, which follows thermal injury to the skin (19). In both models the data support the concept that systemic complement activation leads to pulmonary capillary leukostasis and subsequent PMN-derived $\mathrm{H}_{2} \mathrm{O}_{2}$ mediated endothelial injury. Recently, Ward et al. have provided evidence that $\mathrm{HO}^{\circ}$ may be at least in part responsible for this injury (22). Using the CVFtriggered model of acute lung injury, it has been shown that pretreatment of animals with $\mathrm{HO}^{\prime}$ radical scavengers (dimethylsulfoxide) or iron chelators (human milk apolactoferrin, deferoxamine mesylate) results in significant protection. The apparent requirement for ionic iron to be present for maximal lung injury, together with the previous observation that lung injury can be suppressed with catalase, suggests that the injury may be dependent on $\mathrm{HO}^{\circ}$ formation from $\mathrm{H}_{2} \mathrm{O}_{2}$ via the Fenton reaction (Table 1). The complement activation models of acute lung injury bear particular relevance to our understanding of adult respiratory distress syndrome (ARDS).

Whereas these studies have focused on the role of neutrophil-derived oxygen metabolites in tissue injury, there is increasing evidence that other phagocytes (monocytes, macrophages) may be important effector cells in oxyradical-mediated tissue injury. Macrophages have been implicated as oxyradical generating effector cells in several models of immune complexinduced glomerulonephritis and in recently described models of acute lung injury (9). Johnson et al. have described acute and progressive lung injury in rats after intratracheal administration of phorbol myristate acetate (PMA) (10). Both phases of this lung injury appear to be oxyradical-mediated, since catalase protects against acute and chronic tissue injury. Intratracheal instillation of PMA results in full development of lung injury in PMN-depleted animals, thereby implicating macrophages (alveolar, lung interstitial) or recruited monocytes as critical effector cells.
A second oxyradical-mediated acute lung injury model that implicates mononuclear phagocytes rather than neutrophils involves the in situ formation of immunoglobulin A-containing immune complexes within alveolar septae (11). This lung injury is complement-dependent and neutrophil independent. Subsequent studies have demonstrated that IgA immune complex-induced lung injury can be suppressed by pretreatment of animals with SOD, catalase, deferoxamine, or DMSO (12). In contrast to macrophages harvested by bronchoalveolar lavage (BAL) from rats receiving $\operatorname{IgA}$ into the airways in the absence of intravenously injected antigen, BAL macrophages from rats developing IgA immune complex-mediated lung injury are increased in number, spontaneously generate more $\mathrm{O}_{\overline{2}}^{-}$per cell than controls, and generate more $\mathrm{O}_{\overline{2}}^{-}$per cell after stimulation with PMA. These studies provide convincing but indirect evidence that mononuclear cells can mediate tissue injury.

The body of in vivo and in vitro data, in conjunction with studies documenting oxyradical production by phagocytes, not only provide extensive evidence for the roles of oxygen-derived metabolites in tissue injury but also provide insight into the complexity of pathogenetic mechanisms that exist in the acute inflammatory response.

\section{References}

1. Babior, B. M. (1978). Oxygen-dependent microbial killing by phagocytes. N. Engl. J. Med. 298:659-668, $721-727$.

2. Carp, H. and A. Janoff (1979), In vitro suppression of serum elastase inhibitory capacity by reactive oxygen species generated by phagocytosing polymorphonuclear leukocytes. J. Clin. Invest. 63:793-797.

3. Fantone, J. C. and P. A. Ward (1982). Role of oxygen-derived free radicals and metabolites in leukocytedependent inflammatory reactions. Am. J. Pathol. 107:397-418.

4. Fligiel, S. E. G. et al. (1984). Protein degradation following treatment with hydrogen peroxide. Am. J. Pathol. 115: 418-425.

5. Fridovich, I. (1976). Oxygen radicals, hydrogen peroxide and oxygen toxicity, pp 239-277. In W. A. Pryor (ed.) Free 
radicals in biology, Vol. 1. Academic Press, Inc., New York.

6. Greenwald, R. A. (1979). Oxygen radicals and connective tissue. J. Rheumatol. 8(2): 185- 196.

7. Grgaard, B. et al. (1982). Effects of ischemia and oxygen radicals on mucosal albumin clearance in intestine. Am. J. Physiol. 242:448-454.

8. Johnson, K. J. and P. A. Ward (1981). Role of oxygen metabolites in immune complex injury of lung. J. Immunol. 126:2365-2374.

9. Johnson, K. J. and P. A. Ward (1982). New concepts in the pathogenesis of immune complex induced tissue injury. Lab. Invest. 47(3):218-233.

10. Johnson, K. J. and P. A. Ward (1982). Acute and progressive lung injury with phorbol myristate acetate. Am. J. Pathol. 107:29-35.

11. Johnson, K. J. et al. (1984). Acute lung injury in rat caused by immunoglobulin A immune complexes. J. Clin. Invest. 74:358-369.

12. Johnson, K. J. et al. Mediation of $\lg \mathrm{A}$ induced lung in the rat: role of macrophages and reactive oxygen products. Lab. Invest., in press.

13. Klebanoff, S. J. (1980). Oxygen metabolism and the toxic properties of phagocytes. Ann. Intern. Med. 93: $480-489$.

14. McCormick, J. R. et al. (1981). The effect of superoxide dismutase on pulmonary and dermal inflammation. Am. J. Pathol. 102:55-61.

15. Perez, H. D. and I. M. Goldstein (1980). Generation of a chemotactic lipid from arachidonic acid by exposure to a superoxide generating system. Fed. Proc. 39:1170-1174.

16. Romson, J. L. et al. (1983). Reduction of the extent of ischemic myocardial injury by neutrophil depletion in the dog. Circulation 67:1016-1023.

17. Root, R. K. and M. S. Cohen (1981). The microbicidal mechanisms of human neutrophils and eosinophils. Rev. Inf. Dis. 3:565-598.

18. Till, G. O. et al. (1982). Intravascular activation of complement and acute lung injury. Dependency on neutrophils and toxic oxygen metabolites. J. Clin. Invest. 69:1126-1135.

19. Till, G. O. et al. (1983). Oxygen radical dependent lung damage following thermal injury of rat skin. J. Trauma 23:269-277.

20. Valentine, J. S. (1979). The chemical reactivity of superoxide anion in aprotic versus protic media: a review, pp 659-677. In W. S. Caughey (ed.) Biochemical and clinical aspects of oxygen. Academic Press, Inc., New York

21. Varani, J. et al. (1985). Pulmonary endothelial cell killing by human neutrophils. Possible involvement of hydroxyl radical. Lab. Invest. 53:656663.

22. Ward, P. A. et al. (1983). Evidence for the role of hydroxyl radical in complement and neutrophil-dependent tissue injury. J. Clin. Invest. 72:789-799.

23. Willson, R. L. (1979). Hydroxyl radicals and biological damage in vitro: what relevance in vivo?, pp 19-42. In Oxygen free radicals and tissue damage, Ciba Foundation Symposium 65. Excerpta Medica, Amsterdam.

\section{Suppression of Human Natural Killer Activity by Delta-9- Tetrahydrocannabinol}

\author{
Steven Specter, Ph.D. \\ Department of Medical Microbiology and \\ Immunology \\ University of South Florida College of \\ Medicine \\ Tampa, Florida
}

The adverse effects of chronic marijuana use have been the subject of a considerable number of studies. Psychoactive as well as physiologic and biochemical effects of this drug are well-documented $(4,12)$. The effects of marijuana on the immune system are less complete and highly equivocal. A number of reports indicate that marijuana has detrimental effects on immune responses, suppressing antibody formation $(1,2)$, cell-mediated immunity $(2,9,13)$ and macrophage function $(5,7)$. Conversely, others have reported no effect by marijuana or its components on immunity $(2,20)$. These discrepancies are unexplained, but they are probably due to the different test systems employed in the various studies. Which results most accurately represent chronic marijuana use remain to be determined.

The importance of the above observations has been clarified recently by the suggestion that head and neck cancers in 14 patients are associated with a history of chronic marijuana use but no other common factor. Such cancers are generally seen in patients past $55 \mathrm{yr}$ of age, but these 14 patients were less than 40 yr old (J. Endicott, personal communication). This observation was made by two independent groups (six patients in one study, eight in the other). It is the first suggestion that marijuana use has contributed to a disease process in humans that is related to immunologic competence. The fact that these cancers occur at the site where marijuana is likely to be in highest concentration and most irritating adds to the significance of these observations. Other studies have demonstrated enhanced susceptibility to bacterial and/or viral infections in experimental animals exposed to mari. juana or its components $(2,8,11)$. Thus, we examined more closely the effects of $\delta$-9-tetrahydrocannabinol (THC, the major psychoactive ingredient of marijuana) to determine its in vitro effects on human mononuclear cells isolated from peripheral blood.

\section{THC and Natural Killer Cell Activity}

Initial studies in this laboratory have shown a pronounced effect of THC on natural killer $(\mathrm{NK})$ cell function but little or no effect on $T$ lymphocyte blastogenesis in response to phytohemagglutinin (PHA) or concanavalin A (ConA) (18). Because NK cells are considered to be important in host defenses against tumor cells and microbial agents $(6,14,18)$, this response is being investigated further. In addition, others have shown that chronic injection of THC suppresses NK activity of rat spleen cells (13). Acute exposure (e.g., one injection) had no effect. Studies in mice also indicated NK cell activity is suppressed (T. Klein, personal communication). Furthermore, the mouse studies show that $\gamma$-interferon production in these mice was inhibited by THC. Because interferon is known to boost NK activity $(3,10)$, the authors suggest that the effects of THC 